\title{
Services and Applications Based on Mobile User's Location Detection and Prediction
}

\author{
M. Abo-Zahhad ${ }^{1}$, Sabah M. Ahmed ${ }^{1}$, M. Mourad $^{2}$ \\ ${ }^{1}$ Department of Electrical and Electronic Engineering, Assiut University, Assiut, Egypt \\ ${ }^{2}$ Department of Communication Engineering, Nahda University, Beni Suef, Egypt \\ Email: zahhad@yahoo.com, sabahma@yahoo.com,mohamed.mourad@nub.edu.eg \\ Received February 13, 2013; revised March 18, 2013; accepted April 8, 2013
}

Copyright (C) 2013 M. Abo-Zahhad et al. This is an open access article distributed under the Creative Commons Attribution License, which permits unrestricted use, distribution, and reproduction in any medium, provided the original work is properly cited.

\begin{abstract}
Many wireless applications are deployed and available to customers via their mobile phones. Variety of these applications and services are based on determination of the current or future location of mobile user. Location based services (LBS) are one of the vital applications which are subdivided into two main categories: economical category and public category. Economic applications include mobile marketing, entertainment and tracking applications. Whereas, emergency cases, safety, traffic management, Muslims' applications and public information applications are sort of public applications. The first part of the paper presents a new proposed system with developed procedure to recreate public and economic applications with high positioning accuracy and good authentication of users' data. The developed system is created to enhance both location based services and network allocation resources within mobile network platform using either normal or GPS supported mobile equipment. The second part of the paper introduces future location prediction of mobile user dependent applications. New algorithm is developed depending on utilizing both intra-cell Movement Pattern algorithm (ICMP) [1] and hybrid uplink time Difference of Arrival and Assisted GPS technique (UTDOA_AGPS) [2]. It has been noticed that ICMP algorithm outperforms other future location prediction algorithms with high precision and within suitable time (less than 220) msec. However, UTDOA_AGPS guarantees high precession of mobile user independent of the surrounding environment. The proposed technique is used to enhance reliability and efficiency of location based services using cellular network platform.
\end{abstract}

Keywords: Wireless Location Based Services; Emergency Mobile Applications; Tracking Applications; Muslims' Applications; Future Location Prediction Applications

\section{Introduction}

As a result of great advances in mobile communication systems and due to widely usage of mobile equipments everywhere, there is urgent and increasing demand on mobile applications and services. About 5.6 billion mobile phones are used all over the world within 2011 with percentage $79.68 \%$ of total world population [3]. So, mobile based applications and services are highly required as they reach for most of mobile users without additional hard efforts or extra equipments. Mobile location applications based on basic cellular phones depend on available network platform and there is no need for additional resources unlike smart phones which use extra resources such as WAP, WiFi and internet applications which consume more resources, time and they need additional network infrastructure.

On the other hand, about $28.73 \%$ of total world popu- lations are Muslims. So, Muslims' applications are highly required to support more than quarter of world's population especially when they travel to other countries. Location based services are one of the most growing services based on mobile techniques which play an important role in many life branches. Emergency cases, safety issues, traffic management, Muslim's applications and public information are public type of mobile location services. Marketing, advertisement, entertainment and tracking applications are classified as commercial part of mobile based services.

According to the diagram of Figure 1 about May 11, 2012 report of Pew research center, it is observed that users of location based services with basic cellular phones increased from 28\% in May 2011 to 46\% in February 2012 and users of location based services with smart phones increased from $55 \%$ to $74 \%$ in the same period. According to these results, there are two questions 


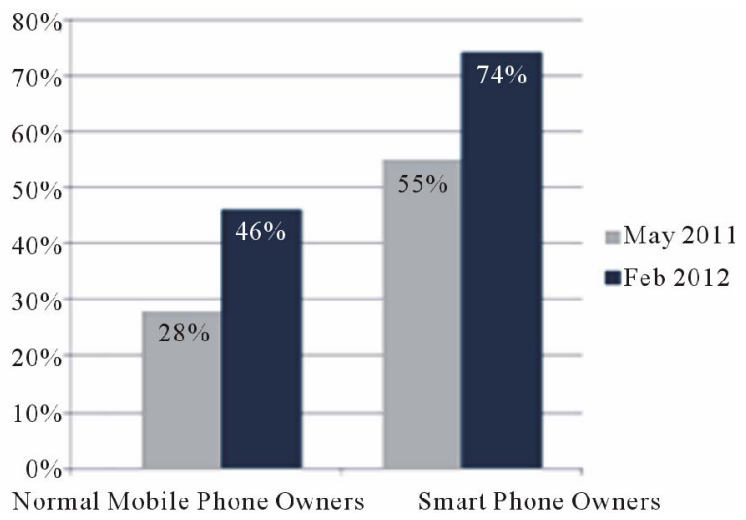

Figure 1. Location based services usage, report of Pew research center.

appeared here.

1) Why there is a great increment in the number of users of location based services and applications?

2) Why the percentage of smart phone owners using LBS is greater than basic cellular phones owners using the same services?

Actually, this section is mainly concentrated on the answer of the above two questions. Due to the progress of wireless location detection technologies from one day to another and as a result of a variety of services and applications based on mobile locations, number of LBS users increased by $20 \%$ within six months. About $90 \%$ of mobile subscribers worldwide use basic mobile phones, so the proposed paper concentrated on location applications over basic mobile phones. Therefore, most of mobile users can utilize location based services and applications smoothly without changing their handsets or paying more and more to get such applications on smart phones.

Smart phones mainly are built on mobile operating systems, with more advanced computing capability and connectivity than basic phones. So, most of LBS and its applications can be executed over these operating systems such as Google's Android, Apple's iOS, Nokia's Symbian, RIM's BlackBerry OS, Samsung's Bada, Microsoft's Windows Phone, Hewlett-Packard's webOS, and embedded Linux. About 458 million working smart phones in 2011 according to Gartner, the world's leading information technology research and advisory company, are used all over the world with percentage less than $10 \%$ of basic cellular phones in use. So, developing basic cellular phones to support location based services will serve $90 \%$ of mobile users all over the world without large costs or demand for new cellular phones with smart technologies and high prices. There are three main entities of positioning process: users, network providers and application service providers [4]. Each of these entities participates in positioning application process according to the proposed system.

In the paper, mobile applications and network advan- tages based on prediction of mobile user's future location are studied. Users, mobile network providers and service providers are able to benefit from mobile location applications. Users' authentication protocols and tracking permissions are designed in the proposed detection system as created in [5]. The paper is organized as follows. Section 1 presents this introduction. Section 2 contains the related publications. System architecture which is used in positioning applications is introduced in Section 3. Public applications based on mobile location are presented in Section 4 with Muslims' applications as an example. Economic applications based on mobile location are regenerated in Section 5. Section 6 shows applications of future location prediction in both mobile network enhancement and users services. Section 7 concludes the paper.

\section{Related Work}

Many researches were developed to detect the position of mobile user depending on cellular phone type and mobile network architecture. Hybrid positioning techniques are used to achieve high positioning accuracy with low time latency. One of the best hybrid positioning techniques is the hybrid Uplink Time Difference of Arrival (UTDOA) and Assisted GPS (A-GPS) technique which has been introduced in [1]. Some enhancements to regular location detection are developed in [5] by verifying user location using location proofs mechanism. Location proofs are time stamped allowing the recipient device to store location data and use them later in the case when an application wants to verify a device's location at some point in the past. In [6] a new and important application is based on web architecture for Muslim travelers with emphasis on social networking. This method introduces some Muslims applications like Qibla direction, prayer time and halal food, permissible food for Muslims, places based on web platform whereas; in this paper most of these applications are created based on mobile network platform.

In [7], privacy study of location based services is discussed. Location-tracking services that are based on other parties tracking the user's location and position-aware services that rely on the device's knowledge of its own location are discussed. One application is the exchange of favors, such as errands that someone else could possibly do in your place if they are closer to a location than you are is mentioned in [8] but it based only on GPS technique. On the other hand, many algorithms were developed to predict the future location of mobile subscriber. In [9], future location prediction algorithm based on Markov model with two-dimensional, time stamped location information to predict future locations is outperformed by ICMP technique generated in [2]. In [10], future location prediction algorithm based on LZ family algorithms was executed over mobile terminals. This 
technique uses GSM architecture for position detection which leads to low positioning accuracy (from $100 \mathrm{~m}$ in urban areas to few kilometers in rural areas). The calculation processes of future prediction over this technique are executed into mobile terminal itself with low memory and less processor than network processors. These limitations make it difficult to perform complex algorithm with high accuracy. In our proposed system, hybrid technique is used to get high accuracy of mobile location depending on network infrastructure and its equipments to calculate the accurate position with modified ICMP algorithm to get the most accurate predictions.

\section{The Proposed System Architecture}

According to Figure 2, the proposed system architecture consists of the following elements:

- Application server;

- Database server;

- Location Gateway (LG);

- Serving Mobile Location Center (SMLC);

- Location Measurement Unit (LMU);

- Base Transceiver Stations (BTS);

- Mobile Station (MS);

- GPS supported Satellites "optional". In the following, two scenarios are presented depending on mobile terminal specifications.

- Non-Supported GPS Mobile terminal: In this case UTDOA technology is used to detect the position of mobile user. SMLC calculates mobile position and sends this data to mobile station with accuracy less than 50 meters.

- Supported GPS Mobile terminal: Hybrid uplink time difference of arrival with assisted GPS technology is used to detect mobile terminal position. In this case both GPS satellites and base stations of mobile network participate in positioning process. SMLC calculates the final position and sends this data to mobile station with accuracy from 5 to 30 meters.

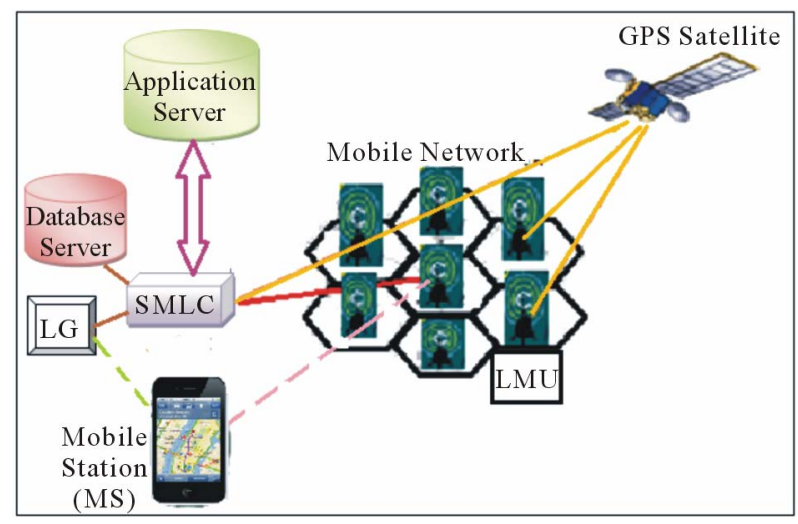

Figure 2. Overall architecture of positioning system.
In the proposed system architecture shown in Figure 2, the application server has dual connection ways to SMLC. The connection between SMLC and application server is based on application type. Application server requests the current position of mobile terminal from the serving SMLC which responds by the accurate position after authentication and confirmation processes. Database (DB) which is associated with the SMLC will record positions of mobile terminals and save results for applications that need position history. LG is connected to both SMLC and MS where it is intermediate part between SMLC and MS. On the other hand, there are some applications based on mobile user request as emergency calls and some Muslims applications. So, mobile device will send request via $L G$ to SMLC which forwards this request to application server to complete services according to application type. The general procedure of detecting mobile location using hybrid positioning technique follows the below steps:

1) MS sends request to LG including serving base-station, cell ID, associated frequency, code, communication parameters and authentication.

2) $\mathrm{LG}$ receives the location request from $\mathrm{MS}$, check authorization and validation.

3) LG sends the request to the SMLC which serves the MS and controls the entire positioning process, including allocation of resources, evaluation of timing measurements, and calculation of position fixes.

4) SMLC receives the request and select the nearest LMUs for serving MS from a list of LMUs included in SMLC database. The database contains the latest data of satellite locations and motion parameters related to each LMU.

5) LMU receives GPS navigation message periodically and extract Doppler shifts, pseudo ranges and navigation messages, correction data and sends these data to SMLC.

6) SMLC receives satellite data from LMU and periodically or on demand evaluates satellites' locations and updates current list of assisting satellites' IDs, Doppler shift and pseudo range domains appropriate for each LMU.

7) SMLC sends the determined GPS data to LG which redistribute its location parameter to MS to be able to detect satellite rapidly and decrease TTFF.

8) SMLC requests location related data from all LMUs selected to cooperate in providing measurements.

9) LMU responds to SMLC by the extracted, measured and obtained data related to the MS of interest.

10) LG receives the GPS data measured by the MS GPS receiver and sends them to the SMLC.

11) SMLC calculates the optimal integration of MS GPS data with the LMU measurements which represent the most accurate location of MS and send it to LG.

12) LG receives location determination from SMLC and sends it to the MS as a response of original location 
request.

The following key goals are achieved from the proposed design.

a) Preserve location privacy of the users while using mobile location applications. So, all data shared in the design are encrypted.

b) The design is flexible to support a variety of location-based applications. We demonstrate this flexibility by using the proposed design to sketch the implementation of several and different types of location-based applications.

c) The proposed design is simple and practical to suite its adaption.

\subsection{System Privacy Rules}

As positioning and tracking services allow service providers to determine and track mobile user location, authentication process and privacy system should be enrolled in the proposed system. A study of security solutions to enable location-based services without using a trusted third party technique due to drawbacks of trusted third party is introduced in [5]. There are two scenarios of location based services. Firstly, services which directly transfers user location information to authorized entities, and thus the technical challenge is to protect the location information from unauthorized entities, including the service provider itself. The second type of service does not involve transfers of user location itself, but it requires computations that take user locations as inputs. In both cases location information is being stored on application server, encrypted using a key specifically chosen for the subset of entities that are authorized to retrieve the location information. Of course, this encrypted location information will be periodically updated. The basic idea of our privacy-preserving design is that only the authorized and permitted application should be able to derive the key to decrypt the location information using cryptographic encryption technique which is motivated by Akl and Taylor's work on hierarchical access control and Fiat and Naor's work on broadcast encryption [5]. This encryption technique proved a good solution to limited spectrum and energy of wireless communication.

\subsection{Database Architecture}

Database server is a type of database warehouse that integrates data from several sources into structures expressly designed for analytical purposes as in [11]. DB is connected to SMLC which is connected to application server directly and to MS via LG and mobile network. DB consists of dimensions and each dimension is organized into a hierarchical structure of levels, which enables the aggregation of facts to the desired levels of granular- ity. DB schema has the following data as shown in Figure 3.

1) Location dimension: contains users' current location at specific timestamps. Two detection levels according to application needed.

- Longitude and latitude coordinates as a first level of detection.

- Cell, sector and sub-sector as a second detection level.

2) Time dimension: consists of specific time of location detection in hh:mm:ss format.

3) Date dimension: consists of year, month and day levels.

4) Content dimension: contains application type according to required data, e.g. information, news, pictures, etc.

5) User dimension: users are classified according to the trusted application servers.

\subsection{Position Calculation}

According to the mobile terminal specification there are two main position calculation techniques.

\subsubsection{Position Calculation Using U-TDOA Technique}

This type of measurement is based mainly on the difference between two Times of Arrivals (TOA) for two distinct signal receptions at least. MS is a common transmitter for signal to the distinct and different LMUs as receiving stations positioned at locations XR1 and XR2 respectively. TDOA technique is directly related to the difference in TOA at different receivers. This leads to difference in signal propagation distances (DR1, and DR2) at receivers.

$$
\mathrm{TDOA}_{21}=\mathrm{TOA}_{2}-\mathrm{TOA}_{1}=\left(\mathrm{DR}_{2}-\mathrm{DR}_{1}\right) / 2 .
$$

From Equation (1) only two LMUs are required to calculate mobile location using UTDOA technique. UTDOA is uniquely suited for mission-critical safety and

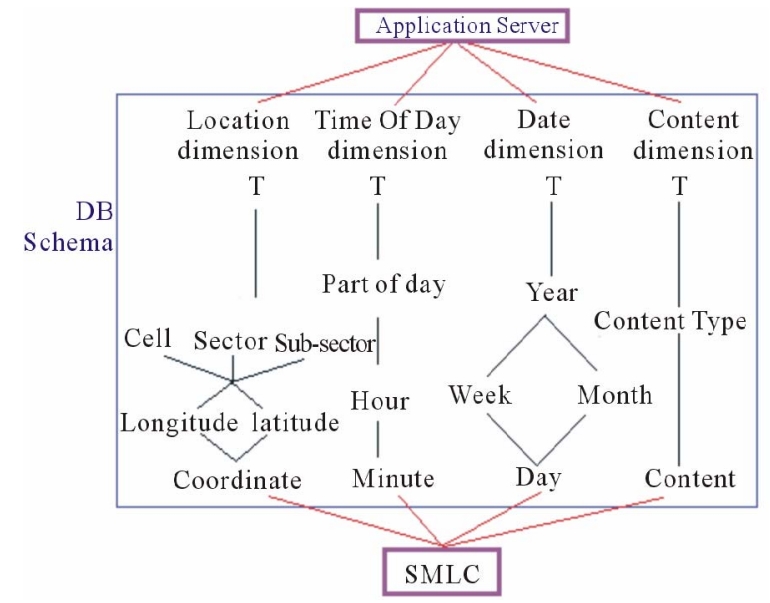

Figure 3. Database warehouse schema in LBS. 
security applications due to:

- Locate mobile phones with very high accuracy.

- Locate mobile phones with very high reliability.

- Locate all mobile phones, even those that are not GPS-enabled, the location capability cannot be disabled by the mobile phone user in any way.

- Locate mobile phones in any environment (urban, suburban, rural, open country, whether indoors, outdoors, or in-vehicle, etc.).

\subsubsection{Position Calculation Using Hybrid Positioning Technique}

The optimum estimation of mobile user location depends on the location-related information available which is extracted from the following sources:

1) Measurement of received signal characteristics.

2) Collateral information that indicate the relative probability of MS position.

Using Bayes probability relation the relative probability of occurrence of measurements under condition of a priori state condition is expressed as:

$$
P(x / z)=[P(z / x) * P(x)] / P(z)
$$

where $x$ is the state vector of location parameters, $z$ is a vector set of location measurements and $P(x / z)$ represents the probability of the state vector components evaluated for $x$ under condition that the observations have the values of measurement values $z$. Whereas $P(z / x)$ represents the probability that the values of vector $z$ would be observed under condition that the state variables are of the values in $x . P(x)$ is the marginal probability that the state values of $x$ occurred. Whereas, $P(z)$ is the total probability of occurrence of measured parameter values for the observation vector $z$. The jointly combined probability of independent data elements are the product of the probability of independent data sets alone [12]. Using the proposed system, the data of various types from diverse sources, satellite and mobile net-work, integrates statistically independent data. Then, the probability product relation is accumulated as a sum of "log likelihood". Sum of probabilities will introduce higher location probability than product formula and higher results can be obtained. This way leads to accumulation and integration between both GPS and UTDOA techniques to get higher positioning technique than obtained from standalone technique. The following advantages are obtained using hybrid technique:

- Reduction of TTFF by utilizing assisted data from LMU in mobile network.

- High positioning accuracy than that obtained by standalone GPS or UTDOA.

- Number of needed GPS satellites is less than that needed in conventional GPS as discussed according to two/three dimension applications.
- The optimal positioning technique such as GPS, UTDOA or hybrid is obtained depending on the positioning parameters such as accuracy, latency, call state, environment and system loading.

\section{Public Applications}

There are a lot of vital public applications in human life as emergency, safety, traffic management, Muslims' applications and public information applications. These public applications serve peoples with common and important needed data and services. Finding places like nearest restaurant, hotel, stations, permissible food for Muslims are most required location services. In this section, some applications are regenerated according to the proposed system.

\subsection{Emergency Applications}

Emergency application is regarded as one of the most important applications which are based mainly on position detection. In many emergency cases, there is no time to describe your location or maybe you are in the high way and you can't describe your position accurately. So, using mobile detection system will solve this problem utilizing the proposed system created in Figure 2. The quality of emergency applications depends on position accuracy and time response. While mobile user requests emergency service, mobile terminal automatically requests position using generated system in [1]. The estimated position will be stored in database server which will send it to emergency server in time. By this technique, time and accuracy are guaranteed in emergency applications.

\subsection{Muslims’ Applications}

Due to travelling from one place to another, there are many services needed by Muslims to complete their religion activities and duties. Moreover, the numbers of Muslim travelers for work or leisure, both domestic and outbound, has increased tremendously. Qibla direction is one of the most important services needed for Muslims to pray. Prayer time which is a basic part of Muslims' life is based mainly on user location and it differs from one location to another even they are in the same country. Many researches introduce public and Muslims' applications based on internet network which need more time, resources and internet supported mobile devices to obtain the required application [6], whereas the proposed system depends on the available mobile network and the regular mobile devices without significant time delay.

\section{Calculation of Qibla's Direction}

Ka'aba, in Makka city, is the destination of Muslims' 
people while praying. So, the direction of Ka'aba is necessary for Muslims' to practice their religious rituals. Ka'aba has the following coordinates: longitude (x-direction): $21^{\circ} 25^{\prime} 20^{\prime \prime}$, latitude (y-direction): $39^{\circ} 49^{\prime} 34^{\prime \prime}$. This coordinates are stored in database server at content dimension. According to [1], SMLC calculates the actual coordinates of mobile device (x1, y1). Ka'baa's direction will be calculated in SMLC using spherical model which is simple and applicable method for bearing or azimuth calculation. This is due to its symmetry where every point on the surface is equidistant from the center. Bearings or azimuths start with 0 degrees toward true north, 90 degrees east, 180 degrees south, and 270 degrees west (clockwise rotation). A great circle is just like every other circle with the additional constraint that its center lies at the center of the sphere. So, lines of longitude are great circles whereas, lines of latitude aren't great circles except equator line. Lines of latitude are in fact circles with their centers lying along the polar axis of the Earth, not necessarily at the center of the Earth. So laws of Cosines and Sines for spherical triangles do not apply to lines of latitude. For great circle paths, the start and end points as well as all the points along the path and the center of the Earth lie in the same plane as shown in Figure 4.

Starting with law of cosine of spherical triangle with $(a, b, c)$ sides of triangle and $(\alpha, \beta, \gamma)$ are its angles we have:

$$
\cos (b)=\cos (a) * \cos (c)+\sin (a) * \sin (c) * \cos (\beta)
$$

where, $\beta=\operatorname{lon} 2-\operatorname{lon} 1, c=\pi / 2-$ lat $1, a=\pi / 2-$ lat 2 .

Substituting these values in (3) leads to:

$$
\begin{aligned}
& \cos (b)=\cos (\pi / 2-\text { lat } 2) * \cos (\pi / 2-\text { lat } 1) \\
& +\sin (\pi / 2-\text { lat } 2) * \sin (\pi / 2-\text { lat } 1) * \cos (\operatorname{lon} 2-\text { lon } 1)
\end{aligned}
$$

Solving for angular distance between the two points (b) yields,

$$
\begin{aligned}
& b=\arccos \{\cos (\pi / 2-\text { lat } 2) * \cos (\pi / 2-\text { lat } 1) \\
& +\sin (\pi / 2-\text { lat } 2) * \sin (\pi / 2-\text { lat } 1) * \cos (\operatorname{lon} 2-\text { lon } 1)\}
\end{aligned}
$$

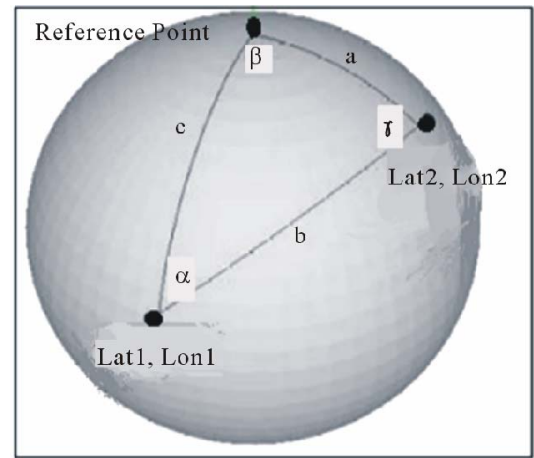

Figure 4. Circle path from point 1 to point 2.
The azimuth from (lat1, lon1) to (lat2, lon2) is calculated by the law of sines:

$$
\begin{aligned}
\sin (\alpha) / \sin (a) & =\sin (\beta) / \sin (b) \\
& =\sin (\gamma) / \sin (c)
\end{aligned}
$$

Then,

$$
\sin (\alpha)=\sin (a) * \sin (\beta) / \sin (b)
$$

Consequently,

$$
\alpha=\arcsin \{\sin (\pi / 2-\text { lat } 2) * \sin (\operatorname{lon} 2-\operatorname{lon} 1) / \sin (b)\}
$$

As shown in Figure 5 and by solving system of equations from (1) to (6), the angle " $\alpha$ " represents the required azimuth between the two points. So, while user is located at point 1 and Ka'baa is located at point 2, the calculated azimuth “ $\alpha$ ” represents the Qibla's direction.

\section{Commercial Applications}

Due to enormous use of mobile devices all over the world, there are many economic and commercial applications and services based mainly on mobile location. Many surveys predict billions of dollars in revenues for mobile advertising [13]. Mobile network operators are well positioned to take up a significant percent of this advertising revenue as they negotiate deals with content providers. Recent deals between media companies, advertising agencies and Internet/software industry also demonstrate significant optimism for future growth [14]. Mobile marketing, gaming and tracking applications represent economical part of mobile positioning services. There are many challenges that should be overcome to achieve good services. Quality of Service (QoS) can be expressed in terms of location accuracy required, response time, and reliability of operation [15].

\subsection{Mobile Marketing Application}

In order to spread commercial advertisements to specific users, mobile operators mainly use cell ID method as positioning system. This method especially in macro cells which serve big area leads to lose of time, money

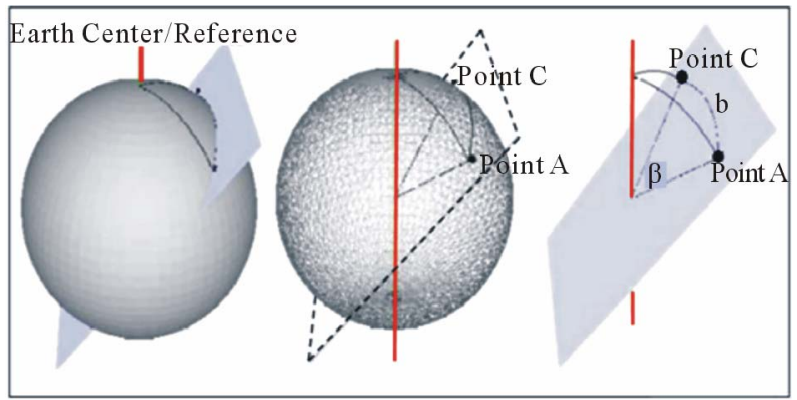

Figure 5. A rectangular planes intersecting a great circle path and the center of the earth. 
and network resources as services may reach to unexpected and undesired customers. Utilizing hybrid uplink time difference of arrival and A-GPS method generated in [1] the accuracy of position is refined. Using equations from 3 to 5 the angular distance between the user and the target is determined.

Arc_length $=$ radius $*$ angular distance

So the distance $(d)$ can be obtained from:

$$
\begin{aligned}
& d=\text { Earth Radius } * \arccos \{\cos (\pi / 2-\text { lat } 2) \\
& * \cos (\pi / 2-\text { lat } 1)+\sin (\pi / 2-\text { lat } 2) \\
& * \sin (\pi / 2-\text { lat } 1) * \cos (\operatorname{lon} 2-\text { lon } 1)\}
\end{aligned}
$$

Using Equations (9) and (10) the distance d is the actual distance between the two points. According to marketing application, server determines the critical distance " $d_{0}$ ". If $d$ is less than or equal $d_{0}$, the user will receive the marketing service. Otherwise, mobile user isn't the target for this service.

\subsection{Finding Places}

One of the most useful applications used nowadays especially in travelling abroad is finding places using mobile phones. The nearest restaurant, mosque, church, cinema, university and any other place can be found using the proposed system. Data base server related to each SMLC contains the location "longitude, latitude" of places and is divided according to each base station. There are two options of finding places: find nearest place or specific place.

1) While user requests the location of nearest place, database server check for the place within the user base station then the neighbor base stations. Location "longitude, latitude" of the place is compared to user location. The distance between user and place can be calculated according to Equations (3)-(5), (8) and (9). Also the direction of the target is determined according to Equations (6) and (7).

2) While the user demands specific location, database server check for the place's location regardless of the base station serving this place. By the same way, the distance between the user and place can be calculated using SMLC according to Equations (3)-(5), (8) and (9). Also the direction of the target is determined according to Equations (6) and (7).

\section{Future Location Prediction}

The progression from location-awareness to locationprediction can enable the next generation of proactive, context-predicting applications. There are many applications depend on the future location prediction to get full the best usage and results. Tracking applications, traffic management services and some military and police re- quirements depend on future location prediction. The mobile phone itself may also be aware of users' future location, thus being able to interact with that location (e.g., an office or home) so it is prepared somehow when the user gets there (computer, lights or heat turned on) [10]. Intra-cell movement pattern (ICMP) algorithm presented in $[2,16]$ is one of the most accurate ways to predict the future location of mobile user. The first step of ICMP algorithm is to determine the current location of mobile device. Therefore, the proposed system utilizes the high accuracy of hybrid uplink time difference of arrival and A-GPS technique to detect the current location of mobile user.

The precision of ICMP algorithm according to predefined confidence value is shown in Figure 6, which indicates good precision that reaches $99 \%$ at high confidence. The precision value is almost $90 \%$ according to change in minimum support (Min. sup) value of ICMP algorithm as shown in Figure 7.

\subsection{Process of Future Location Prediction}

According to our system presented in Figure 2, the current position of mobile subscriber is obtained. By utilize ing the Intra-Sector Prediction (ISP) level of ICMP algorithm presented in [2]. As the latitude and longitude of mobile user are obtained in accurate manner with the proposed hybrid technique, the needed data for ISP method will be in the form of [Pmn, Xi, Yi, Ti]. Where, (Pmn) represents Cell ID $(\mathrm{m})$ and sector ID $(\mathrm{n}),(\mathrm{X}, \mathrm{Y})$ represent user coordinates (latitude and longitude respecttively) and $\mathrm{T}$ is the time set of measurement. By applying the following mechanisms, a high predicted

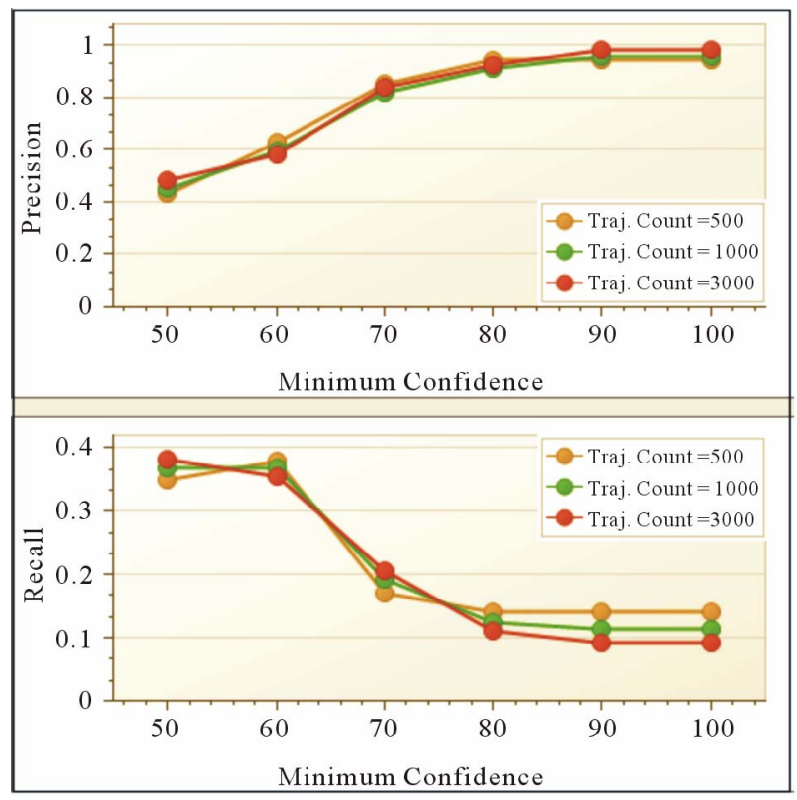

Figure 6. Precision and recall versus confidence value. 


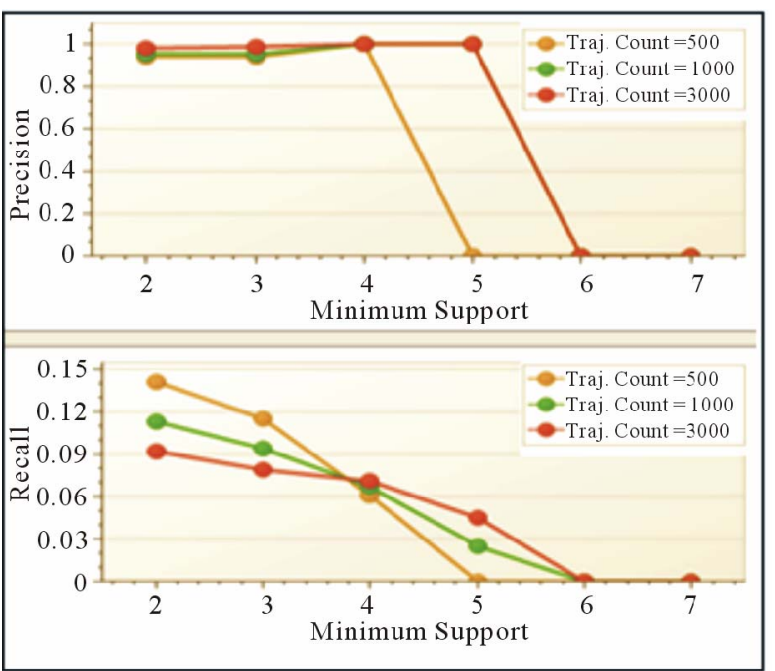

Figure 7. Precision and recall versus min. support value.

future location is obtained.

1) Moving sequences' creation process.

2) Extraction of all frequent movement patterns.

3) Finding movement rules.

4) Prediction of user's next movement.

5) Finding moving sequences.

According to the flow chart indicated in Figure 8, the output of this process is a high accurate predicted mobile location within timestamp. Accuracy of this model is depending upon the required confidence which depends on the needed application specs. Also, the next cell which will serve the user in the future is determined which enhance allocation resources and availability of network service. So, both mobile network resources and location based services are enhanced depending on the proposed prediction technique.

\subsection{Traffic Management Application}

When there are more crowds in some streets, road administrators need to turn the way to another road or warn cars' users. By using location prediction technique, application server related to road administration will send the warning messages to users whose future locations are in the target road. The confidence threshold value is adjusted according to the required prediction accuracy. Also, for more precise applications when there is a need to inform the user by distance or direction of specific target like stop point, crowded point, etc. equations from (3) to (10) is used. This technique achieves good quality of service due to good adjustable accuracy, less time delay and save money and resources of mobile network operators.

\subsection{Children, Elders and Friend Tracking Applications}

In this section, a new and valuable application is gener-

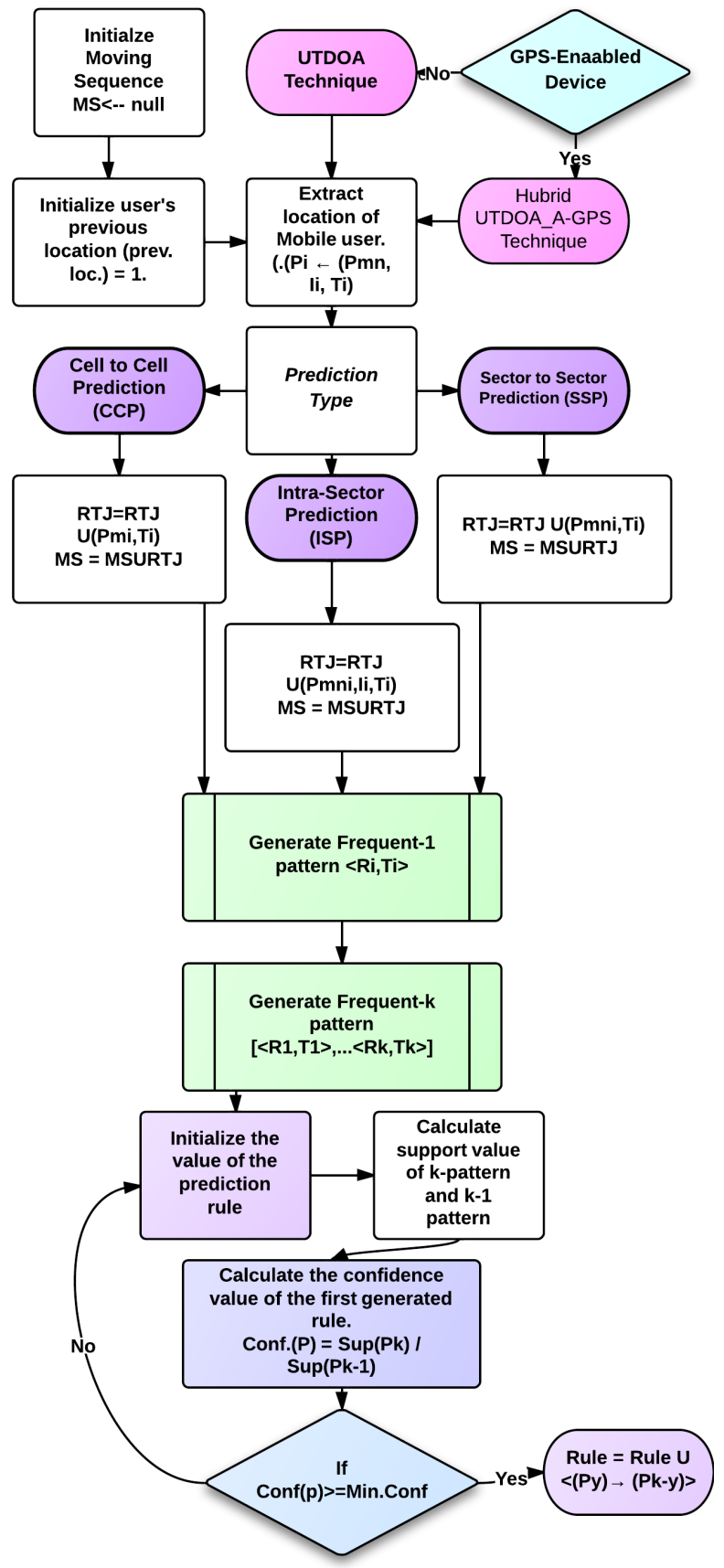

Figure 8. Flow chart of proposed future location prediction based on ICMP algorithm.

ated. Using short message service mechanism, parents can be informed whether their children lost their path to school or didn't reach their expected destination in predicted time. Parent phone or any other people responsible of children phone number are stored in database related to child number. By applying the proposed prediction algorithm it is possible to predict a child (x) who should reach a specific location within time (Tx). So if this prediction achieved then, short message is sent to parents' mobile phone that user (x) already reach the predicted 
target. Otherwise, alarm message to parent phone is reached that child $(\mathrm{x})$ didn't reach the predicted target. By the same way older people are tracked by their healthcare persons or friends. In this application, user privacy mechanism introduced in [7] is used to control which cellular phones is accepted to track others and authentication behavior. All the above data should be stored in application server which is connected to SMLC and mobile network to achieve the tracking application properly.

\section{Conclusions}

Location-based services have attracted considerable attention due to their potential to transform mobile communications and the potential for a range of highly personalized and context-aware services. In this paper, many services and applications based on the detection of mobile location are regenerated according to accurate hybrid positioning technique with mobile network system including database server. The proposed positioning technique has the following advantages:

- High position accuracy, less than $50 \mathrm{~m}$ precession with low time latency especially at time to first fix (TTFF).

- High flexibility as various mobile networks and GPS topologies are used to detect mobile location.

- Database server enables history of position data which is used at many applications.

- Privacy and authentication for users' locations.

- Future location prediction with high accuracy, precision and in small time depending on ICMP algorithm.

Emergency applications, Qibla direction as one of the most important Muslims applications and mobile marketing are based on the proposed system. The proposed technique is applicable on basic mobile phones without any hardware addition. Mobile operators and application service providers can achieve high revenues due to location services. On the other hand, future location predicttion is highly effective to enhance mobile network allocation resources, hand over process and mobility management operations. Future location prediction applications and services as traffic management and tracking applications are based on ICMP algorithm with hybrid positioning technique to achieve highest quality of location services.

\section{REFERENCES}

[1] M. Abo-Zahhad, S. M. Ahmed and M. Mourad, "Hybrid Uplink-Time Difference of Arrival and Assisted-GPS Positioning Technique," International Journal of Communications, Network and System Sciences, Vol. 5, No. 6, 2012, pp. 303-312. doi:10.4236/ijens.2012.56040

[2] M. Abo-Zahhad, S. M. Ahmed and M. Mourad, "Map
Based Intra-Cell Method for Location Prediction over UMTS Network Platform," JES Journal, Vol. 39, No. 5, 2011, pp. 1069-1094.

[3] "List of Countries by Number of Mobile Phones in Use," Wikipedia, Gartner, 2010. http://www.gartner.com /it/page.jsp?id=1759714

[4] R. Kokku, K. Sundaresan and G. F. Jiang, "Enabling Location Specific Real-time Mobile Applications," MobiArch'08, Seattle, 2008, pp. 19-24.

[5] S. Zhong, E. R. Li, Y. B. G. Liu and Y. R. Yang, "Privacy-Preserving Location-Based Services for Mobile Users in Wireless Networks," Yale Computer Science Technical Report YALEU/DCS/TR-1297, 2004.

[6] T. S. Gunawan, M. Kartiwi and A. A. F. C. Omar, "Development of Web Application for Muslim Traveller with Emphasis on Social Networking," ICCIT Conference, 2628 June 2012, pp. 1-12.

[7] L. Barkuus and A. Dey, "Location-Based Services for Mobile Telephony: A Study of Users' Privacy Concerns," 9th IFIP TC13 International Conference on Human-Computer Interaction, 9 July 2003, pp. 1-5.

[8] D. Ashbrook and T. Starner, "Using GPS to Learn Significant Locations and Predict Movement across Multiple Users," Personal and Ubiquitous Computing, Vol. 7, No. 5, 2003, pp. 275-286. doi:10.1007/s00779-003-0240-0

[9] I. E. Burbey, "Predicting Future Locations and Arrival Times of Individuals," Ph.D. Research, Virginia University, Virginia, 2011.

[10] A. Rodriguez-Carrion, C. Garcia-Rubio, C. Campo, A. Cortés Martín, E. Garcia-Lozano and P. Noriega-Vivas, "Study of LZ-Based Location Prediction and Its Application to Transportation Recommender Systems," Sensors Journal, Vol. 12, No. 6, 2012, pp. 7496-7517.

[11] C. S. Jensen, A. Friis-Christensen, T. B. Pedersen, D. Pfoser, S. Saltenis and N. Tryfona, "Location-Based Services: A Database Perspective," Proceedings of ScanGIS, 2001, pp. 59-68.

[12] R. J. Anderson and J. E. Maloney, "TDOA/GPS Hybrid Wireless Location System," US Patent No. US7925274B2, 2011.

[13] “Analysis: Are Mobile Ads the Next Goldrush?" Wireless News, 2007.

http://wirelessfederation.com/news/category/informa-tele coms/

[14] K. Delaney, "How Yahoo Plans to Get Things Going on the Go", Wall Street Journal, 2007.

[15] S. Dhar and U. Varshney, "Challenges and Business Models for Mobile Location-Based Services and Advertising," Communications of the ACM, Vol. 54, No. 5, 2011, pp. 121-128.

[16] M. Abo-Zahhad, S. M. Ahmed and M. Mourad, "Future Location Prediction of Mobile Subscriber over Mobile Network Using Intra Cell Movement Pattern Algorithm," International Conference on Communications, Signal Processing, and Their Applications, Sharjah, December 14 2012, pp. 1-6. 